\title{
Highly stable and low loss electro-optic polymer waveguides for high speed microring modulators using photodefinition
}

\author{
M. Balakrishnan, M. B. J. Diemeer, A. Driessen, \\ Integrated Optical Microsystems, MESA ${ }^{+}$Institute for Nanotechnology, P.O. Box 217, 7500 AE \\ Enschede, The Netherlands. \\ M. Faccini, W. Verboom, D. N. Reinhoudt \\ Laboratory of Supramolecular Chemistry and Technology, $\mathrm{MESA}^{+}$Institute for Nanotechnology, \\ P.O. Box 217, 7500 AE Enschede, The Netherlands. \\ A. Leinse \\ LioniX BV, P.O. Box 456, 7500 AH Enschede, The Netherlands
}

\begin{abstract}
Different electro-optic polymer systems are analyzed with respect to their electro-optic activity, glass transition temperature $\left(\mathrm{T}_{\mathrm{g}}\right)$ and photodefinable properties. The polymers tested are polysulfone (PS) and SU8. The electro-optic chromophore, tricyanovinylidenediphenylaminobenzene (TCVDPA), which was reported to have a high photochemical stability ${ }^{1}$ has been employed in the current work. Tert-butyl-TCVDPA, having bulky side groups, was synthesized and a doubling of the electro-optic coefficient $\left(\mathrm{r}_{33}\right)$ compared to the unmodified TCVDPA was shown. A microring resonator design was made based on the PS-TCVDPA system. SU8 (passive) and TCVDPA (active) channel waveguides were fabricated by the photodefinition technique and the passive waveguide losses were measured to be $5 \mathrm{~dB} / \mathrm{cm}$ at $1550 \mathrm{~nm}$.
\end{abstract}

Keywords: Photodefinition, electro-optic polymers, grafting process, microring resonators, Electro-optic modulators.

\section{INTRODUCTION}

Over the past few years there has been an increased interest in electro-optic polymers for telecommunication applications. Due to their potential for low drive voltage, integration ability, low optical losses in the 1.3 and $1.55 \mu \mathrm{m}$ telecommunication windows, and low dispersion of refractive index between optical frequencies and millimeter waves, electro-optic polymer modulators are being targeted for $>40 \mathrm{~Gb} / \mathrm{s}$ data rate communication systems. Microring resonator based photonic devices have been investigated extensively in the recent years because of their potential application in highly integrated photonic circuits. The device is typically in the form of a microring closely coupled to a waveguide and offers unique properties like narrow bandwidth filtering, high bandwidth modulation, strong modulation response and compactness.

In this article we present the fabrication of waveguides consisting of passive regions with a serially grafted active section to form a low loss ring resonator device. The use of polymers offers a number of advantages compared to semiconductor based devices. The first one is reduced loss. The surface roughness resulting from dry etching can induce a large scattering loss, which is the main loss mechanism in the fabricated microring devices ${ }^{2}$. Such a high loss places a significant limitation on the practical use of these microring resonator devices. Since the surface roughness is proportional to the refractive index contrast between the waveguide and the cladding, the use of low refractive index contrast polymers will significantly reduce such loss. The losses can further be reduced by the process of direct photodefinition thereby avoiding the use of dry etching processes. Polymers also offer the possibility of reflowing after fabrication to reduce the surface roughness ${ }^{3}$. On the other hand a high refractive index contrast is required for making small rings. With the contrast available in polymers, a ring radius of about $50 \mu \mathrm{m}$ is possible. The second advantage is a better coupling efficiency to optical fibers than previously demonstrated with semiconductor waveguides due to the low refractive index contrast and large crosssection of the polymer waveguides. This will greatly reduce the device insertion loss. The third advantage is the potentially high nonlinearity in polymers which will reduce the switching voltage.

Integrated Optics: Devices, Materials, and Technologies X, edited by Yakov Sidorin, Christoph A. Waechter, Proc. of SPIE Vol. 6123, 61230J, (2006) - 0277-786X/06/\$15 - doi: 10.1117/12.645243 


\section{MATERIALS}

To date several host materials like polymethylmethacrylate (PMMA), polyimide, polyurethane etc., are used for both material and device studies. In the current work we report the usage of the TCVDPA chromophore in two different host materials namely, PS and SU8. Both polymers have a high $T_{g}$ which is required to maintain the alignment of the chromophores after poling. The samples were then poled by heating them close to their $\mathrm{T}_{\mathrm{g}}$ and applying an electric field in the order of $100 \mathrm{~V} / \mu \mathrm{m}$ across the films for 20 minutes. Nonlinear optical properties were evaluated by measuring the $\mathrm{r}_{33}$ coefficient using the Teng-Man reflection technique at $830 \mathrm{~nm}^{4}$ with films on ITO coated glass substrates covered by a gold top electrode. All the $\mathrm{r}_{33}$ values mentioned were measured at $830 \mathrm{~nm}$ because of the ITO absorption at higher wavelengths.

\subsection{PS-TCVDPA}

PS-TCVDPA constitutes the guest-host system in which the TCVDPA chromophore is simply dissolved in the PS host. Films with different concentrations of TCVDPA in PS were prepared and the $r_{33}$ coefficient was measured. The solutions of PS were prepared in cyclopentanone. They were filtered through a $0.2 \mu \mathrm{m}$ filter, spin coated and dried in a vacuum oven at $95^{\circ} \mathrm{C}$ for 8 hours. Figure 1 shows the plot of the $\mathrm{r}_{33}$ versus chromophore concentration expressed in wt $\%$ for TCVDPA and tert butyl-TCVDPA. For the latter it is expressed as an effective wt $\%$ such that for a given wt $\%$ in the polymer the chromophore number density remains the same both chromophores, (for the structures see figure 2).

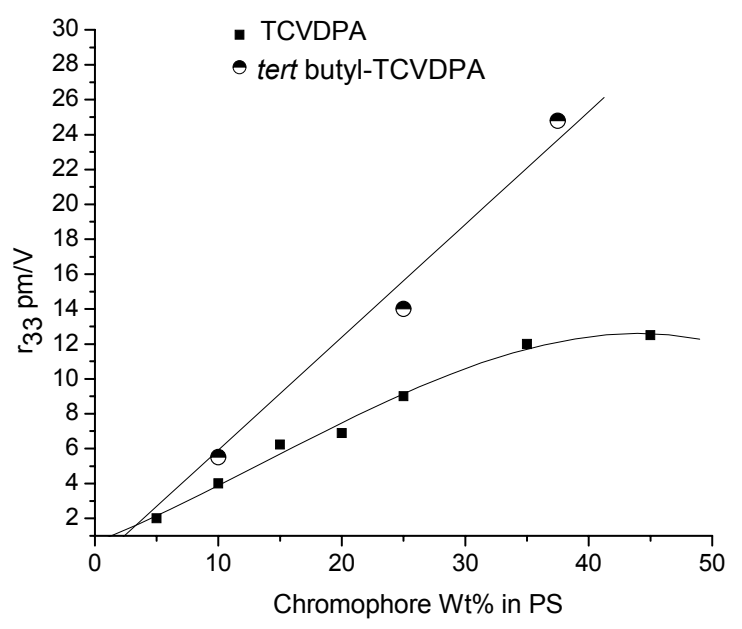

Figure1: Variation of $\mathrm{r}_{33}$ with TCVDPA and tert butyl-TCVDPA concentrations in PS. The concentrations of TCVDPA with tert-butyl group are expressed as effective concentrations of TCVDPA for comparison

From figure 1 it can be seen that in the PS-TCVDPA system, the $\mathrm{r}_{33}$ increases linearly up to a concentration of about 15 $\mathrm{wt} \%$, above which the curve starts to flatten. The maximum $\mathrm{r}_{33}$ measured was $12.5 \mathrm{pm} / \mathrm{V}$. The flattening can be explained by increased intermolecular dipole-dipole interactions between the chromophores causing antiparallel clustering with no contribution to the electro-optic effect. The intermolecular interaction between the chromophores can be reduced by attaching bulky side groups to the chromophores, making them more spherical and thereby preventing their closer approach ${ }^{5}$. Tert butyl-TCVDPA shows a linear increase in $r_{33}$ up to $40 \mathrm{wt} \%$ of the chromophore. The best result was an $r_{33}$ of $25 \mathrm{pm} / \mathrm{V}$, a doubling compared with TCVDPA without side group.

One of the advantages of using polysulfone as host is its high $\mathrm{T}_{\mathrm{g}}$ of about $190^{\circ} \mathrm{C}$. However, the chromophores act as plasticizers and reduce the $T_{g}$ of the host polymer drastically when added as guest (figure 3 ). The $T_{g}$ of the polymer with different concentrations of the chromophores was determined by observing the buckling of a gold layer of $100 \mathrm{~nm}$ thickness sputtered onto a polymer layer on a silicon wafer, as the temperature of the polymer is slowly ramped. Figure 3 shows the $T_{g}$ as a function of the chromophore concentration for the two chromophores. From figure 3 it can also be seen that the chromophore with a lower molecular mass has a higher plasticizing effect. This sets a limit to the maximum achievable chromophore concentration in order to maintain an acceptable $\mathrm{T}_{\mathrm{g}}$. 
<smiles>N#CC(C#N)=C(C#N)c1ccc(N(c2ccccc2)c2ccccc2)cc1</smiles>

a

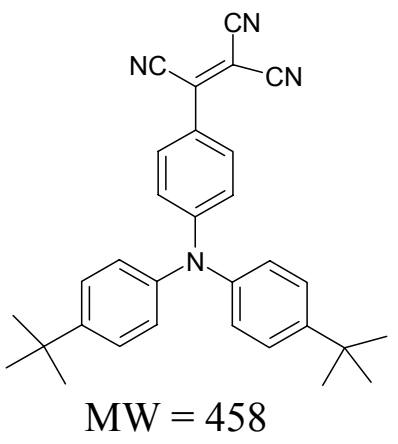

b

Figure 2: Molecular structures of TCVDPA (a), tert butyl- TCVDPA (b) with their molecular weight

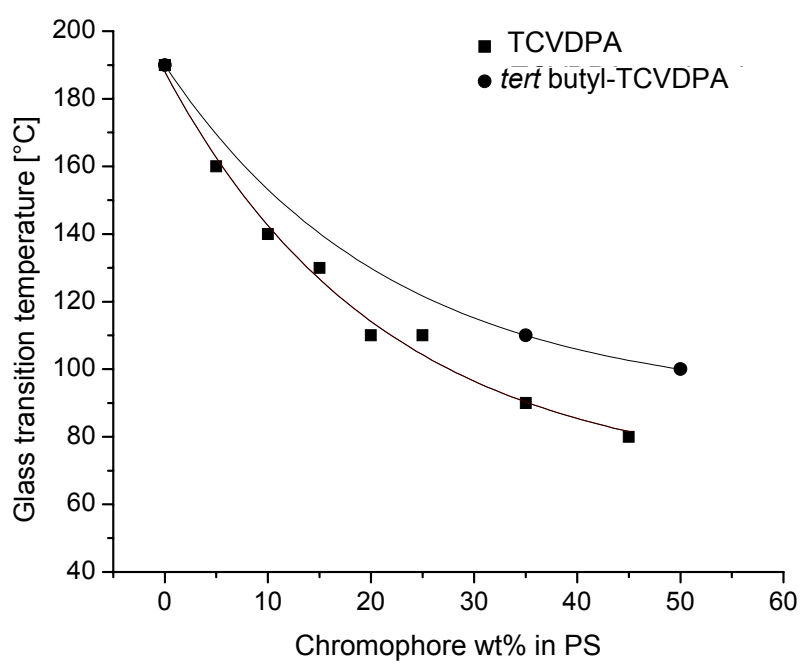

Figure 3: Variation of $\mathrm{T}_{\mathrm{g}}$ with chromophore concentration in PS

\subsection{SU8-TCVDPA}

This system offers the advantage of direct photodefinition of the host negative epoxy photoresist SU8 by exploiting the low UV absorption window of the TCVDPA chromophore ${ }^{6}$. A film with $15 \mathrm{wt} \%$ TCVDPA in SU8 photoresist was spin coated on ITO glass. A soft bake was done at $95{ }^{\circ} \mathrm{C}$ for 5 minutes and UV exposed in a contact mask aligner for 5 minutes. The exposed film was crosslinked by post baking it at $120^{\circ} \mathrm{C}$ for 10 minutes. The film was poled at $50^{\circ} \mathrm{C}$. (The $\mathrm{T}_{\mathrm{g}}$ of the crosslinked film is reduced from about $200^{\circ} \mathrm{C}$ to $50^{\circ} \mathrm{C}$ because of the plasticizing effect of the chromophores. An $\mathrm{r}_{33}$ value of $5.5 \mathrm{pm} / \mathrm{V}$ was measured. The reduction in the $\mathrm{T}_{\mathrm{g}}$ was attempted to be counteracted by crosslinking TCVDPA with epoxy groups to the SU8 polymer. The molecular structures of SU8 and TCVDPA with epoxy groups are shown in figure 4 . The $\mathrm{T}_{\mathrm{g}}$ increased only to $70{ }^{\circ} \mathrm{C}$ in the crosslinked SU8 film equipped with TCVDPA with epoxy groups. The $\mathrm{r}_{33}$ was measured to be $10 \mathrm{pm} / \mathrm{V}$. This increase in $\mathrm{r}_{33}$ can be explained by the bulkiness provided by the epoxy groups. The crosslinking reaction between the TCVDPA with epoxy groups and SU8 has to be investigated more to further increase the $T_{g}$ of this system. 


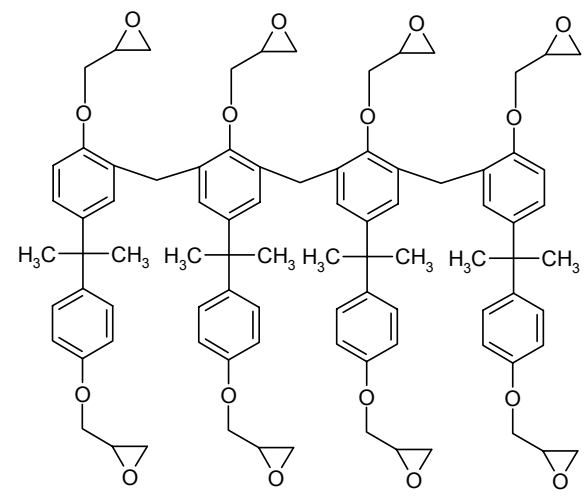

a

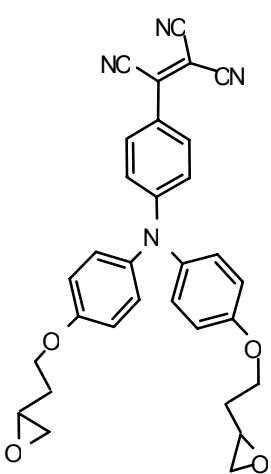

b

Figure 4: SU8 (a) and epoxy-TCVDPA (b) molecules

\section{MICRORING RESONATOR DESIGN}

The microring resonator consists of a ring (diameter: 50 to $500 \mu \mathrm{m}$ ) centered between two waveguides (figure 5). One waveguide is used as the input and through port and the other as the output (drop) port. The straight waveguides are not directly connected to the ring but are positioned such that there is a small gap between the ring and the straight waveguides. Because of this gap only a small fraction of the inbound light is first coupled to the ring. The light that enters the ring then makes half a round trip and a part of the power in the ring is coupled to the drop port. The light then completes its round trip and interferes with the light that enters on the input side. When the phases of the light in the ring and the light entering the ring correspond, constructive interference occurs and there will be buildup of power in the ring. In this so called resonant state of the ring maximum power of a certain wavelength is extracted from the input waveguide and directed into the drop waveguide. When the phases of the light in the ring and the light entering the ring do not match, no constructive interference will occur and therefore the ring will extract hardly any light.

The straight waveguide can lie in the same plane as that of the ring (lateral coupling) or can be stacked vertically (vertical coupling). Both of the schemes have their own advantages and disadvantages. Lateral coupling is easy for fabrication with a single masking step at the expense of tight lithographic control of the coupling gap. In the case of vertical coupling the straight waveguide and the ring can be designed separately while the lateral coupling imposes some design restrictions ${ }^{7}$. The drawback here is the difficult alignment of port waveguides to the microring.

The microring can be made to act as a modulator by electro-optically tuning the refractive index of the ring material. By using electro-optic polymers, a low cost, high speed and small size modulator can be realized. Here we present an unique approach for the modulator fabrication using photodefinable buffer layers combined with a passive SU8 waveguide section and an active PS-TCVDPA section. The microring resonator to be designed is shown in figure 5. Inverted ridges are defined in a photodefinable epoxy buffer layer material, VSC, with a refractive index of 1.5 at $1550 \mathrm{~nm}$ to form the input and output waveguides and the microring. The ridges are then filled with the passive waveguiding material SU8. The SU8 is then partly removed by photodefinition from the region close to the ring and filled with the active material PS-TCVDPA with a refractive index of 1.62 at $1550 \mathrm{~nm}$. The filling of the active region is greatly assisted by the very good planarization property of PS-TCVDPA. This combination of active/passive sections reduces the losses, as the length of the active section with relatively high losses is minimized.

\subsection{Straight waveguide design}

The input and the output waveguides should be monomodal at the operating wavelength. The dimensions of the active region of the input and the output waveguides were simulated by performing $2 \mathrm{D}$ simulation using $\mathrm{C} 2 \mathrm{~V}$ software ${ }^{8}$. Figure 6 shows the monomodal condition for the two polarizations (TE and TM) as a function of the ridge height (RH) and the ridge width (RW). The curve in figure 6 marks the boundary between monomodal and bimodal behavior in addition to 
the effective mode index $\left(\mathrm{n}_{\text {eff }}\right)$ at the boundary points. For dimensions above the curve the waveguide becomes bimodal. As the ring and the waveguides are laterally coupled, the straight ridge waveguide height $(\mathrm{RH})$ and the ring height $(\mathrm{RiH})$ should be the same. The ridge height has to be chosen in such a way that the ridge width is more than $1.5 \mu \mathrm{m}$ so that it can be easily fabricated by contact mask lithography.

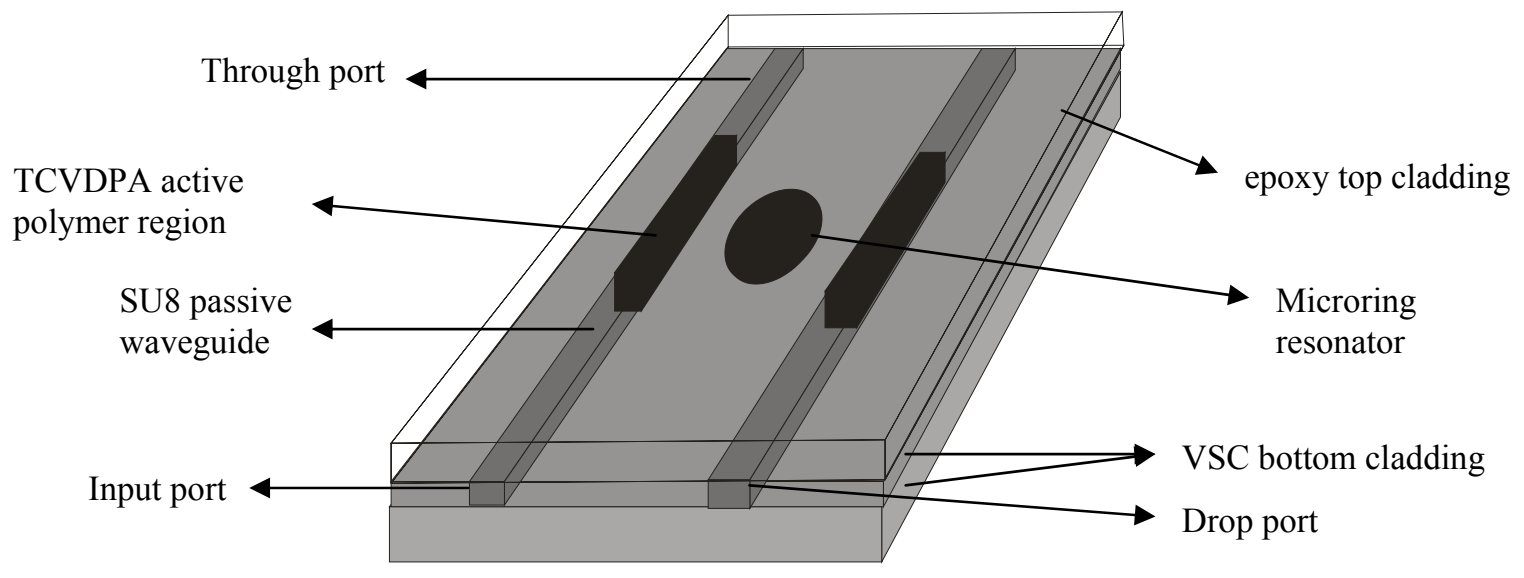

Figure 5: Schematic of an inverted ridge defined in VSC with passive SU8 waveguides and an active TCVDPA polymer region

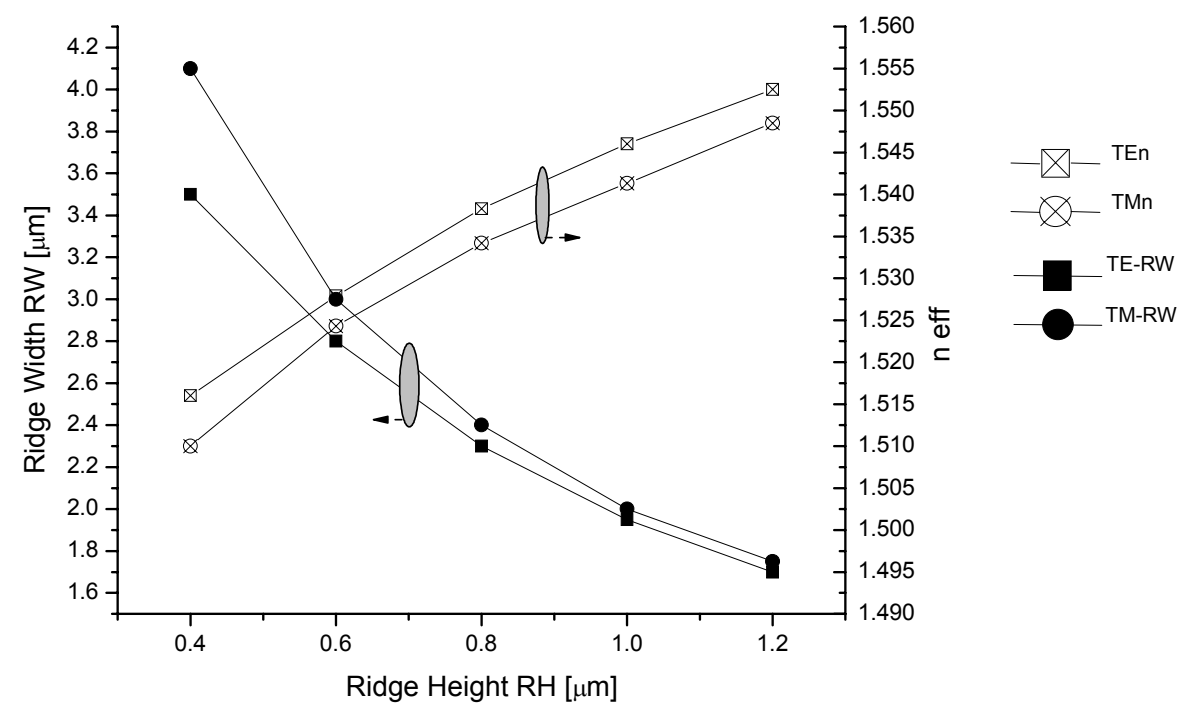

Figure 6: Monomodal condition for the active PS-TCVDPA waveguides with VSC claddings and their $\mathrm{n}_{\text {eff }}$ at the bimodal point

\subsection{Ring design}

Ring losses and the effective index of the bend mode were simulated using the $\mathrm{C} 2 \mathrm{~V} 2 \mathrm{D}$ bend mode solver. The results for the TE and TM polarizations are shown in figure 7. For the ring to be brought in resonance the bend losses should not be more than $1 \mathrm{~dB} / \mathrm{cm}$. It can be seen that as the ring height decreases, the radius of the ring (RR) must increase in order that the bending losses are less than $1 \mathrm{~dB} / \mathrm{cm}$. As the ring radius increases the bending loss decreases but the roundtrip loss increases. This sets the limit for the maximum allowed ring radius. It is also desired to have a small ring radius in 
order to achieve a large free spectral range of the rings. A ring width of $7 \mu \mathrm{m}$ is chosen so that mode field does not sense the other side of the ring (whispering gallery mode to reduce the loss from side wall roughness)

\subsection{Phase matching between the ring and the ridge waveguide}

In order to achieve a maximum coupling between the waveguides and the ring, the effective index of the modes in the ring and the waveguides should match ${ }^{9}$. From figure 6 and 7 the dimensions of the straight waveguide and the ring are chosen in such a way that the effective index of the modes in the straight waveguide and the ring match for bending losses less than $1 \mathrm{~dB} / \mathrm{cm}$. Table 1 gives the dimensions of the ring and the straight waveguide for phase matching. The ring width was chosen to be $7 \mu \mathrm{m}$ in order that the ring mode is in the whispering gallery regime. Figure 8 shows the region where the ring and the straight ridge waveguide can be phase matched for a ridge height of $0.8 \mu \mathrm{m}$ as an example.

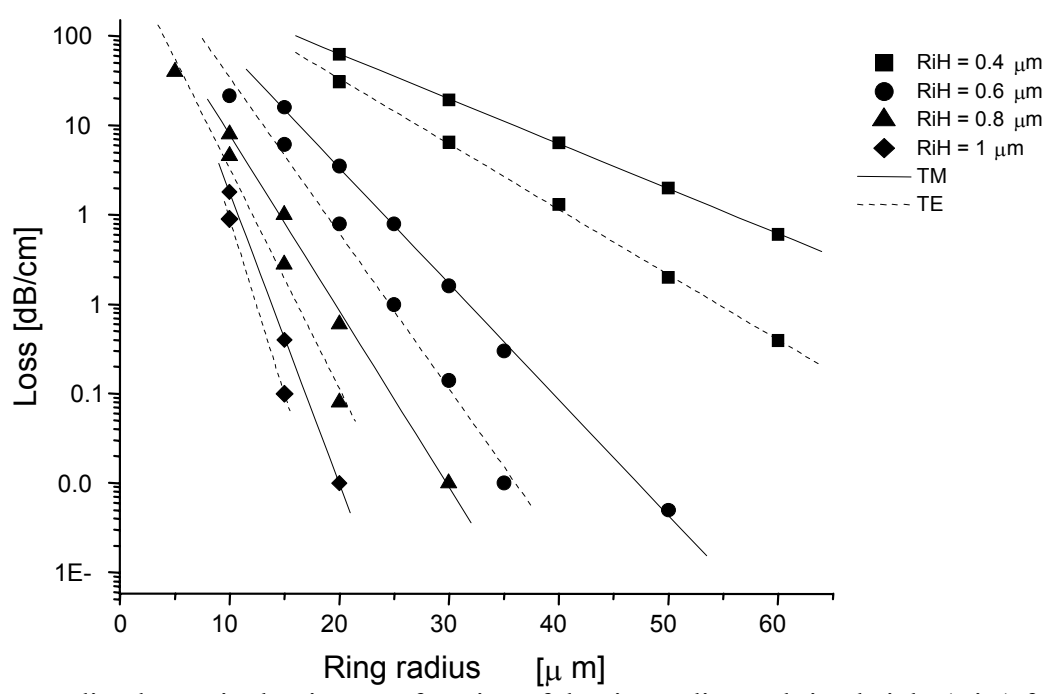

Figure 7: Bending losses in the ring as a function of the ring radius and ring height $(\mathrm{RiH})$ for the TE and TM polarization.

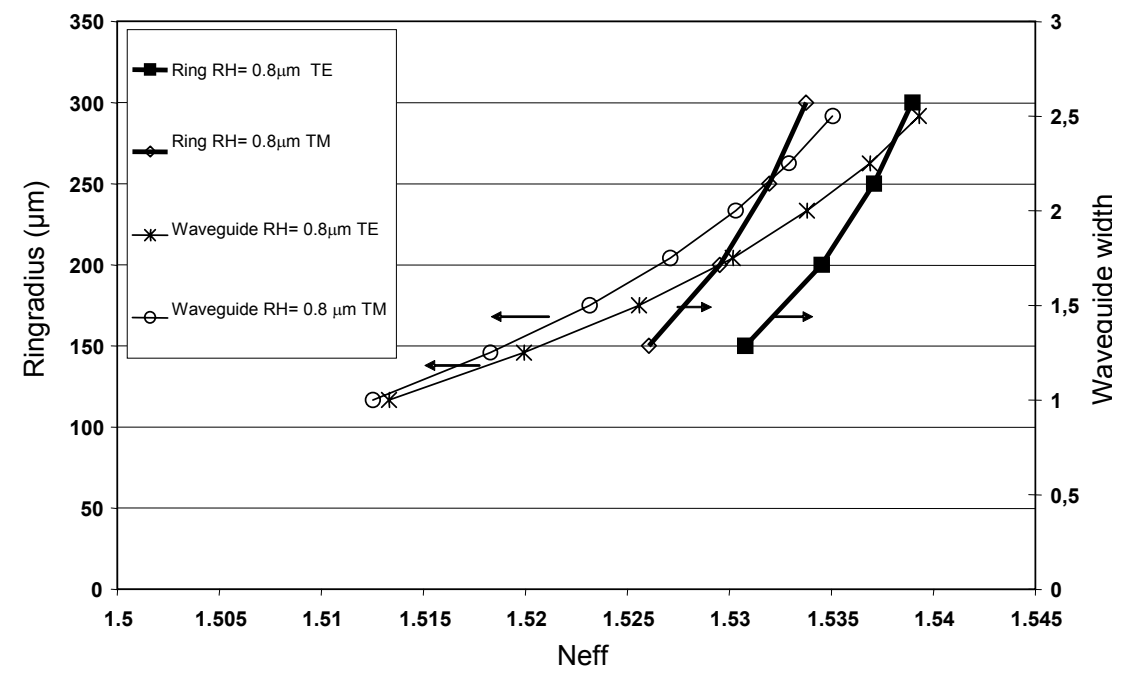

Figure 8: Effective index of the ridge and ring for a ridge height of $0.8 \mu \mathrm{m}$ for the TE and TM polarization. 
Table1:Ring and straight ridge waveguide dimensions for phase matching

\begin{tabular}{|c|c|c|c|}
\hline $\begin{array}{c}\text { Ridge height } \\
\mu \mathrm{m}]\end{array}$ & Ridge width $[\mu \mathrm{m}]$ & Ring radius $[\mu \mathrm{m}]$ & Ring Width $[\mu \mathrm{m}]$ \\
\hline 0.5 & 2.4 & 400 & 7 \\
\hline 0.6 & 2.2 & 300 & 7 \\
\hline 0.8 & 1.9 & 200 & 7 \\
\hline 1 & 1.8 & 150 & 7 \\
\hline 1.2 & 1.5 & 100 & 7 \\
\hline
\end{tabular}

\subsection{Coupling constant}

The coupling constant defines the fraction of the power which couples from the ridge waveguide to the ring. The coupling constant together with the roundtrip losses of the ring determine the quality factor $(\mathrm{Q})$ of the ring. When the waveguide and the ring are phase matched, the coupling constant can be varied monotonically by varying the distance between the ring and the ridge waveguide. Low values of the coupling constant lead to high $\mathrm{Q}$ values, but the on resonance power in the drop port reduces for lossy rings. Therefore it is necessary to strike at the right value of the coupling constant to extract maximum power at the drop port and to have a desired width of the ring response curve. The values of the coupling constants for lateral coupling between the ring and the ridge were simulated by a 3D beam propagation method using $\mathrm{C} 2 \mathrm{~V}$ software. Lateral coupling, though it imposes some design constraints, is more favorable for achieving symmetric coupling than the vertical coupling. Figure 9 shows the coupling between the ring and the ridge for the phase matching dimensions given in table 1. It can be seen from the graph that for a given gap more coupling takes place for a ring of larger radius because of enhanced phase matching as the ring mode profile tends towards that of the straight waveguide for larger ring radii. For a gap of $1 \mu \mathrm{m}$ the power coupling constants are in the range of 0.1 .

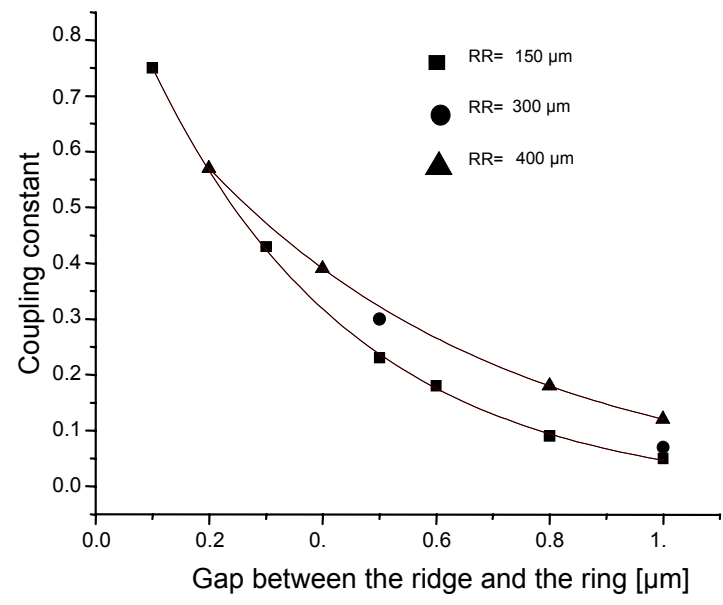

Figure 9: Coupling constants as a function of the gap between the ring and the ridge for different ring radii and TM polarization

In a waveguide coupled microring resonator, a gap larger than $1 \mu \mathrm{m}$ is desirable as it increases the fabrication tolerance. For a given coupling coefficient, the gap size can be enlarged if the coupling distance is increased. The coupling distance can be increased by several configurations. The most obvious is the racetrack configuration where it can be increased by simply extending the length of the straight section. However, this also increases the cavity length. In order to keep the cavity length unchanged, we propose a concentric ring structure (figure 10) in which the microring is coupled to a concentric waveguide curved over a significant part of its circumference. The modal mismatch between the straight and the bend sections which occurs in the racetrack can also be avoided in the concentric structure. If the two waveguides in a concentric structure are identical, the coupling between them will go out of phase because the physical pathlengths in the two waveguides are different. The pathlengths can be matched by varying the widths of the waveguides ${ }^{10}$. 

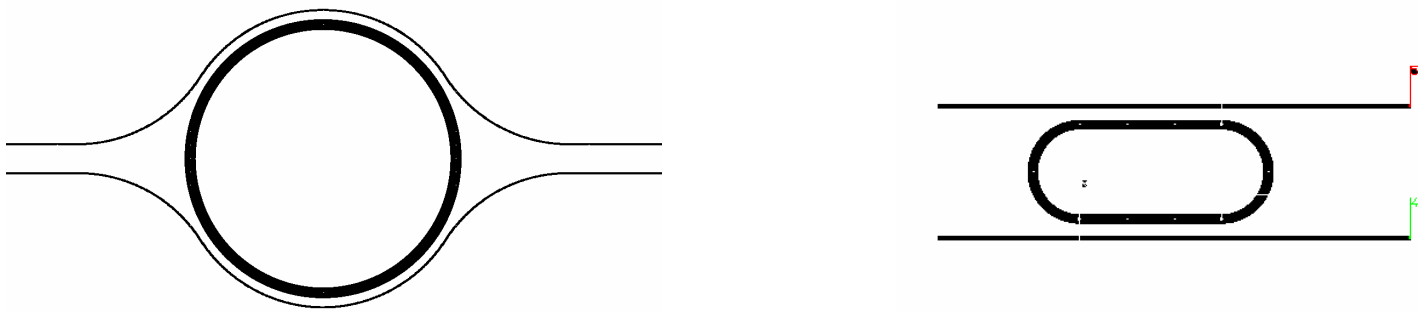

Figure 10: Different coupling schemes, concentric ring (left) and racetrack (right)

\section{FABRICATION}

\subsection{Waveguide fabrication}

Waveguides were fabricated by a combination of reactive ion etching and photodefinition. Firstly, inverted ridges were photodefined in a cladding material (VSC) with a refractive index of 1.5. The photodefined channels were then filled with SU8 as passive waveguide material. Losses in the SU8 waveguides were determined by the cutback technique to be $5 \mathrm{~dB} / \mathrm{cm}$ at $1550 \mathrm{~nm}$. This passive waveguide will be partially removed by photodefinition and replaced with TCVDPA polymer by spin coating, as active material for device fabrication. A SEM picture of a photodefined inverted ridge with the TCVDPA polymer on top is shown in figure 11. The refractive index of SU8 is 1.58 at $1550 \mathrm{~nm}$ and hence losses because of mode mismatch at the active / passive interface are expected to be negligible. The residual slab heights of the active and passive layers will be etched back to zero by reactive ion etching.

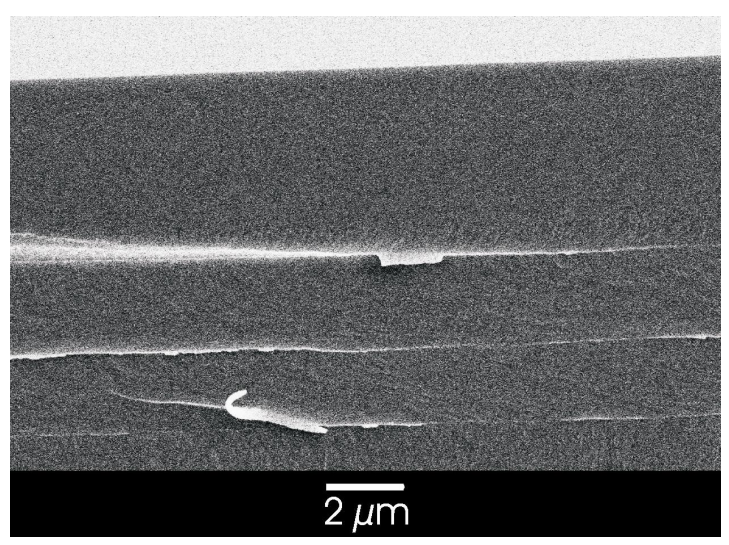

Figure 11: SEM picture of a photodefined ridge in VSC with a PC-TCVDPA layer on top

\section{CONCLUSION}

In conclusion, TCVDPA and its modification were incorporated in PS, and SU8. Tert-butyl TCVDPA is effective in reducing the intermolecular interactions and thereby yielding the highest measured $r_{33}$ value of $25 \mathrm{pm} / \mathrm{V}$. A laterally coupled microring resonator to be used as an electro-optic modulator has been designed and a concentric structure for the coupling to the ring proposed. The electro-optic microring will be grafted into the passive polymer port waveguides to reduce the insertion losses. Ridge waveguides were fabricated by photodefinition and the losses were measured to be 5 $\mathrm{dB} / \mathrm{cm}$ in the SU8 passive waveguides.

This research work is supported by the Dutch Technology Foundation STW, Applied Science Division of NWO and the Technology Program of the Ministry of Economic Affairs and carried out within the framework of the project TOE 6067 "Broadband and wavelength selective modulators for optical communication based on electro-optic polymers". 


\section{REFERENCES}

${ }^{1}$ A. G. Gonzalez, G. I. Stegeman, A. K. Y. Jen, X. Wu, M. Canva, A. C. Kowalczyk, X. Q. Zhang, and H. Z. Lackritz, "Photostability of electro-optic polymers possessing chromophores with efficient amino donors and cyano containing acceptors", J. Opt. Soc. Am., B vol 18, pp 1846-1848, 2001.

${ }^{2}$ V. Van, P. P. Absil, J. V. Hryniewicz, and P. T. Ho, "Measurement and modeling of propagation losses in semiconductor racetrack microresonators", Technical Digest of CLEO 2001, pp 263-264, 2001.

${ }^{3}$ A. Leinse, M. B. J. Diemeer and A. Driessen, "Scattering loss reduction in polymer waveguides by reflowing", IEE Electron. Lett., vol 40, 16, pp 992-993, 2004.

${ }^{4}$ C. C. Teng and H. T. Man, "Simple reflection technique for the measuring the electro-optic coefficient of poled polymers", Appl. Phys. Lett., vol 56, pp 1734-1736, 1990.

${ }^{5}$ B. H. Robinson and L. R. Dalton, "Monte Carlo statistical mechanical simulations of the competition of intermolecular electrostatic and poling field interactions", J. Phys. Chem. A, vol 104, pp 4785-4795, 2000.

${ }^{6}$ B. Murali, A. Driessen, M. B. J. Diemeer, M. Faccini, W. Verboom, D. N. Reinhoudt, A. Borreman, and M. J. Gilde, "Channel waveguide definition in electro-optic polymers with a novel chromophore by UV-thermal crosslinking", Proceedings LEOS Beneulux , pp 76-79, 2004.

${ }^{7}$ A. Leinse, M. B. J. Diemeer, and A. Rousseau, "A novel high speed polymeric electro-optic modulator based on a combination of a microring resonator and an MZI”, IEEE Photon. Technol. Lett., vol 17, 10, pp 2074-2076, 2005.

${ }^{8}$ software@.c2v.nl.

${ }^{9}$ D. H. Geuzebroek, A. Driessen, "Wavelength filters for fiber optics", Editor H. Venghaus, Springer-Verlag, chapter 9, to be published mid 2006.

${ }^{10}$ P. P. Absil, J. V.Hryniewicz, B. E. Little, F. G. Johnson, K. J. Ritter, and P. T. Ho, "Vertically coupled microring resonators using polymer wafer bonding", IEEE Photon. Technol. Lett., vol 13, pp 49-64, 2001. 